ARTICLE

Received 27 Sep 2012 | Accepted 15 Jan 2013 | Published 19 Feb 2013

DOI: $10.1038 /$ ncomms2487

\title{
Influenza neuraminidase operates via a nucleophilic mechanism and can be targeted by covalent inhibitors
}

Christopher J. Vavricka ${ }^{1,2}{ }^{\star}$, Yue Liu ${ }^{2, \star}$, Hiromasa Kiyota ${ }^{3}$, Nongluk Sriwilaijaroen $^{4,5}$, Jianxun $\mathrm{Qi}^{2}$, Kosuke Tanaka $^{3}$, Yan $\mathrm{Wu}^{2}$, Qing $\mathrm{Li}^{2,6}$, Yan $\mathrm{Li}^{2}$, Jinghua Yan${ }^{2}$, Yasuo Suzuki ${ }^{5} \&$ George F. Gao ${ }^{1,2,6,7}$

Development of novel influenza neuraminidase inhibitors is critical for preparedness against influenza outbreaks. Knowledge of the neuraminidase enzymatic mechanism and transitionstate analogue, 2-deoxy-2,3-didehydro- $\mathrm{N}$-acetylneuraminic acid, contributed to the development of the first generation anti-neuraminidase drugs, zanamivir and oseltamivir. However, lack of evidence regarding influenza neuraminidase key catalytic residues has limited strategies for novel neuraminidase inhibitor design. Here, we confirm that influenza neuraminidase conserved Tyr406 is the key catalytic residue that may function as a nucleophile; thus, mechanism-based covalent inhibition of influenza neuraminidase was conceived. Crystallographic studies reveal that $2 \alpha, 3$ ax-difluoro- $N$-acetylneuraminic acid forms a covalent bond with influenza neuraminidase Tyr406 and the compound was found to possess potent anti-influenza activity against both influenza A and B viruses. Our results address many unanswered questions about the influenza neuraminidase catalytic mechanism and demonstrate that covalent inhibition of influenza neuraminidase is a promising and novel strategy for the development of next-generation influenza drugs.

\footnotetext{
${ }^{1}$ Research Network of Immunity and Health (RNIH), Beijing Institutes of Life Science (BIOLS), Beijing 100101, China. ${ }^{2}$ CAS Key Laboratory of Pathogenic Microbiology and Immunology, Institute of Microbiology, Chinese Academy of Sciences, Beijing 100101, China. ${ }^{3}$ Graduate School of Agricultural Science, Tohoku University, Aoba-ku Sendai 981-8555, Japan. ${ }^{4}$ Faculty of Medicine, Thammasat University, Pathumthani 12120 , Thailand. ${ }^{5}$ College of Life and Health Sciences, Chubu University, Aichi 487-8501, Japan. ${ }^{6}$ School of Life Sciences, University of Science and Technology of China, Hefei, Anhui Province 230027 , China. ${ }^{7}$ Chinese Center for Disease Control and Prevention (China CDC), Beijing 102206, China. ${ }^{\star}$ These authors contributed equally to this work.

Correspondence and requests for materials should be addressed to G.F.G. (email: gaof@im.ac.cn).
} 
t has recently been demonstrated that highly pathogenic $\mathrm{H} 5 \mathrm{~N} 1$ avian influenza can easily adapt to become airbornetransmissible in mammals ${ }^{1,2}$, reminding the world about the pandemic potential of avian influenza viruses ${ }^{3,4}$. Moreover, only two drugs, zanamivir and oseltamivir, that function as influenza neuraminidase (NA) inhibitors are currently recommended for use worldwide. Therefore, the development of novel influenza drugs is critical for preparedness against possible future influenza epidemics and pandemics.

The influenza A virus genome comprises 8 negative strand RNA segments encoding 13 proteins ${ }^{5}$. Two major glycoproteins, hemagglutinin (HA) and NA, as well as the M2 ion channel, are on the surface of the virions and are therefore highly accessible targets for drug development ${ }^{6,7}$. Of these three transmembrane proteins, only NA is known to function as an enzyme and is therefore an ideal target for the design of competitive inhibitors that can mimic catalytic transition-state intermediates ${ }^{8}$. Accordingly, influenza NA is currently the most successful drug-target against influenza virus.

Besides NA inhibitors, only M2 ion channel inhibitors have been fully developed; however, the influenza M2 inhibitors amantadine and rimantadine are no longer in use due to serious problems with drug resistance ${ }^{8}$. The design of influenza NA inhibitors is regarded a classical example of rational structurebased drug design, but there are currently only two NA inhibitors, oseltamivir and zanamivir, that are approved for use worldwide ${ }^{8}$. Furthermore, oseltamivir resistance was reported to be highly prevalent in seasonal H1N1 isolates from September 2008 to January $2009^{9}$, whereas zanamivir is not orally active and has a short duration of action. Two more recently developed NA inhibitors, laninamivir and peramivir, are approved for use in Japan and still under phase III clinical trials in the United States. All of these current NA inhibitors were designed based upon the structure of 2-deoxy-2,3-didehydro- $N$-acetylneuraminic acid (Neu5Ac2en), a putative NA transition-state analogue ${ }^{8,10-12}$. Insight into the NA catalytic mechanism and the proposed oxocarbenium ion transition-state intermediate were crucial for the design of Neu5Ac2en analogues with potent NA inhibitory activity $^{13,14}$. However, evidence regarding the key influenza NA catalytic residues has remained elusive and the successful design of highly effective next-generation influenza NA inhibitors has proven to be a difficult task.

Influenza NA is a retaining glycosidase that cleaves the $\alpha$-ketosidic bond of terminally linked sialic acid, notably $\mathrm{N}$-acetylneuraminic acid (Neu5Ac), the predominant sialic acid in humans ${ }^{8}$. A majority of known retaining glycosidases utilize a pair of conserved active-site carboxylate residues with one functioning as a catalytic nucleophile in a double-displacement mechanism ${ }^{15,16}$. Retaining NA mechanisms, on the other hand, appear to be quite distinct. Biochemical analysis of NA from various microorganisms has revealed that a conserved active-site tyrosine can function as a nucleophile and may be the most important catalytic residue ${ }^{17-19}$.

Although influenza NAs share very little sequence identity with NAs from other microorganisms, their active sites are highly conserved, which suggests that they all may function in a similar manner ${ }^{8}$. Leaving group effect and kinetic isotope effect experiments with influenza NA carried out by Guo et al. ${ }^{16}$ and Chou et al. ${ }^{20}$ are compatible with the classical mechanism that includes a covalent enzyme-sialosyl intermediate. Studies by Burmeister et al. ${ }^{21}$ and Ghate et al. ${ }^{22}$ also suggest that Tyr406 has an important role in the influenza NA mechanism. However, numerous studies still indicate that the influenza NA may possibly function in a unique manner. For example, 4-guanidinoNeu5Ac2en (zanamivir), 4-amino-Neu5Ac2en and even Neu5Ac2en all exhibit selectivity for influenza NA over NA from other species ${ }^{10}$; a computational study by Thomas et al. ${ }^{23}$ concluded that a direct hydroxylation mechanism with no covalent influenza NA-sialosyl intermediate is actually the energetically preferred pathway; moreover, some bacterial NAs that possess the same canonical protein fold and conserved active-site tyrosine still produce completely different products ${ }^{16,24}$. Taken together, the above studies clearly illustrate that the influenza virus NA catalytic mechanism may be quite unique. Therefore, experimental evidence is necessary to determine whether the influenza NA conserved active-site tyrosine is really a key catalytic residue and whether covalent inhibition of influenza NA is even possible.

In this study, we first confirmed the catalytic role of the conserved active-site tyrosine (Tyr406, N2 numbering is used throughout the text) of influenza NA in order to explore the possibility of developing covalent influenza NA inhibitors. Influenza N2 was used as a classical example in this study, as $\mathrm{N} 2$-containing viruses are among the most common seasonal strains. Furthermore, the pathogenic agents of the 1957 H2N2 pandemic (Asian flu) and $1968 \mathrm{H} 3 \mathrm{~N} 2$ pandemic (Hong Kong flu) are both $\mathrm{N} 2$-containing influenza $A$ viruses. NMR and crystal soaking experiments clearly demonstrated that substitution of N2 Tyr406 with aspartic acid results in a switch of the influenza NA catalytic mechanism from a retaining glycosidase mechanism to an inverting mechanism. This further indicated that Tyr406 likely functions as a catalytic nucleophile; therefore, 2 $\alpha$,3ax-difluoroNeu5Ac was synthesized and explored as a potential covalent influenza virus NA inhibitor. Soaking of N2 crystals with $2 \alpha, 3 \mathrm{ax}-$ difluoro-Neu5Ac produced the first influenza virus NA covalent complex structure, proving that influenza NA Tyr406 functions as a catalytic nucleophile and that covalent NA inhibition is indeed possible. Most importantly, the covalent inhibitor $2 \alpha, 3$ ax-difluoro-Neu5Ac achieved potent inhibition against both influenza $A$ and $B$ viruses as well as oseltamivir-resistant His274Tyr-NA.

\section{Results}

Tyr406 is the key catalytic residue of influenza NA. Although Ghate et al. ${ }^{22}$ were successful with the analysis of Tyr406Phe NA from influenza $\mathrm{B}^{22}$, a previous report by Lentz et al. ${ }^{25}$ demonstrated that the phenylalanine 406 substitution actually results in an aberrantly processed influenza A N2 with loss of NA activity. Therefore, we chose a different approach by using a baculovirus expression system to produce recombinant $\mathrm{N} 2$ (rN2) from pandemic A/RI/5 + /1957(H2N2) with a Tyr406Asp substitution. Substitution of Tyr406 with aspartic acid yielded a stable protein (rN2-Tyr406Asp) that was purified to homogeneity. rN2-Tyr406Asp had 100-fold reduced $\mathrm{K}_{\text {cat }}$ and two-fold reduced $\mathrm{K}_{\mathrm{m}}$ as shown using kinetic analysis with a standard 4-methylumbelliferyl-Neu5Ac-based fluorescent assay (Table 1). This confirms that Tyr406 is a critical factor for the catalytic activity of influenza NA. The enzymatic reaction of $\mathrm{rN} 2$ and rN2-Tyr406Asp was further monitored with ${ }^{1} \mathrm{H}$ NMR using the avian influenza receptor analogue $3^{\prime}$-sialyllactose. According to previous studies confirming that influenza NA is a retaining

\section{Table 1 | Fluorescence-based kinetic analysis of wild-type influenza rN2 compared with rN2-Tyr406Asp.}

\begin{tabular}{lcc} 
Enzyme & $\boldsymbol{K}_{\mathbf{m}}(\boldsymbol{\mu} \mathbf{M}) \mathbf{( 9 5 \%} \mathbf{~ C l )}$ & Relative $\boldsymbol{K}_{\text {cat }}{ }^{*}$ \\
\hline rN2 & $46.5(40.1-53.0)$ & $100 \pm 2$ \\
rN2-Tyr406Asp & $103.2(87.0-119.4)$ & $1.11 \pm 0.02$ \\
\hline${ }^{*}$ The absolute $K_{\text {cat }}$ value for $W T$ is $21.3 \pm 0.4 \mathrm{~s}^{-1}$. & \\
\hline
\end{tabular}


glycosidase ${ }^{13}$, wild-type $\mathrm{rN} 2$ initially produced $\alpha$-Neu5Ac, which mutarotates to form the more stable $\beta$-Neu5Ac over time. Interestingly, the $\beta$-anomer of Neu5Ac was detected as the initial product of $3^{\prime}$-sialyllactose after incubation with rN2-Tyr406Asp, which demonstrates that rN2-Tyr406Asp has switched from a retaining to inverting glycosidase mechanism (Fig. 1a and b).

A novel $\beta$-Neu5Ac-influenza NA complex structure. rN2Tyr406Asp was then crystallized and crystal soaking experiments were performed using $3^{\prime}$-sialyllactose. Despite its impaired catalytic activity, rN2-Tyr406Asp still hydrolysed the avian receptor analogue during soaking, resulting in the first influenza NA$\beta$-Neu5Ac complex structure, at a resolution of $1.50 \AA$ (Fig. 2b). Crystallographic data and refinement statistics are shown in Table 2. Free sialic acids are much more stable in their $\beta$ anomeric configuration in solution; nevertheless, Neu5Ac always binds to wild-type NA as the $\alpha$-anomer in a boat conformation 8 (Fig. 2b). The additional space created by substitution of Tyr406 with a smaller aspartic acid residue allows for the Neu5Ac to adopt a $\beta$-conformation where the $\mathrm{C}$ - 2 hydroxy group in the $\mathrm{N} 2$ Tyr406Asp- $\beta$-Neu5Ac complex occupies the same region as the Tyr406 hydroxyl group of the wild-type complex. Furthermore, substitution of Tyr406 with Asp406 creates space for additional two water molecules between the C-2 hydroxyl group and Asp406 side-chain (Fig. 2b). Taken together, these results reveal that Tyr406 is indeed the key influenza NA catalytic residue and

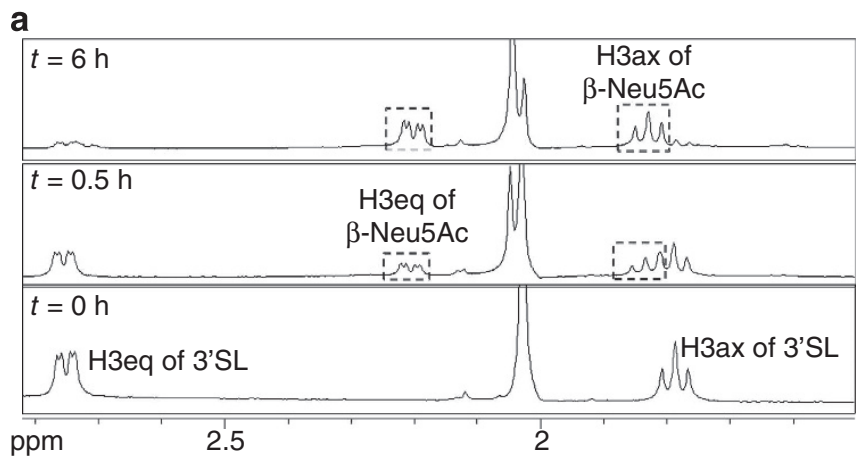

b

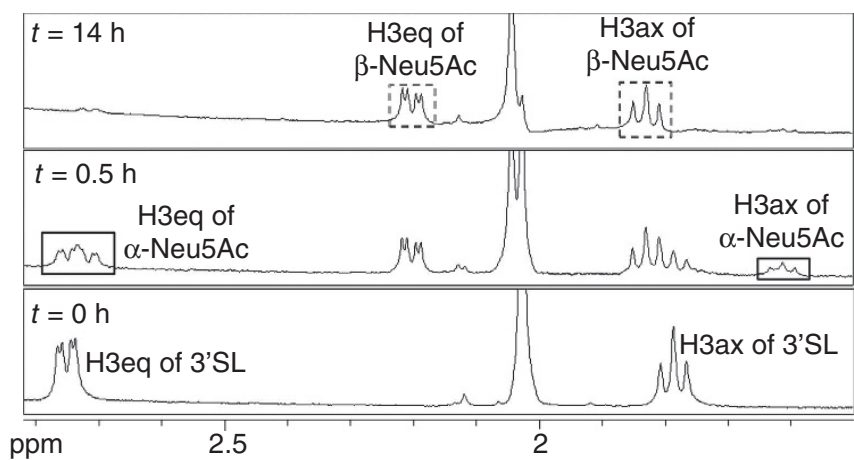

Figure 1 | Conserved Tyr406 is the key catalytic residue of influenza neuraminidase. (a) ${ }^{1} \mathrm{H}$ NMR analysis shows the initial and continuous production of $\beta$-Neu5Ac after incubation of $\mathrm{rN2}$-Tyr406Asp with avian influenza receptor analogue $3^{\prime}$-sialyllactose, demonstrating that rN2-Tyr406Asp has shifted to an inverting glycosidase mechanism. (b) Wild-type influenza NA, in this case rN2, follows a retaining mechanism and therefore initially produces $\alpha-\mathrm{Neu} 5 \mathrm{Ac}$ from 3 -sialyllactose; the $\alpha$-Neu5Ac undergoes mutarotation to form the more stable anomer, $\beta$-Neu5Ac, over time. suggest that Tyr406 likely functions as a nucleophile that can be targeted by covalent inhibitors.

Targeting influenza NA Tyr406 with a covalent inhibitor. Based on our biochemical and structural analysis regarding the conserved Tyr406, we hypothesized that there was a high probability of targeting influenza virus NA with covalent inhibitors. In order for Tyr406 to undergo nucleophilic attack of the Neu5Ac anomeric carbon, a good leaving group should replace the C-2 hydroxyl group ${ }^{26}$. Another electronegative group at the C-3 position should also be present to destabilize any oxocarbenium ion transition-state intermediate, thereby stabilizing the potential Tyr406-inhibitor covalent bond ${ }^{26}$. Fluorinated compounds have been investigated for their anti-NA activity for quite some time and are excellent choices for covalent influenza NA inhibitors ${ }^{26-30}$. Although 2 3 ,3eq-difluoro-Neu5Ac, which has been explored as an inhibitor of parainfluenza virus, was readily available from our previous studies ${ }^{30}$, structural analysis clearly demonstrates that the compound should be in the $\alpha$-anomeric configuration so that the
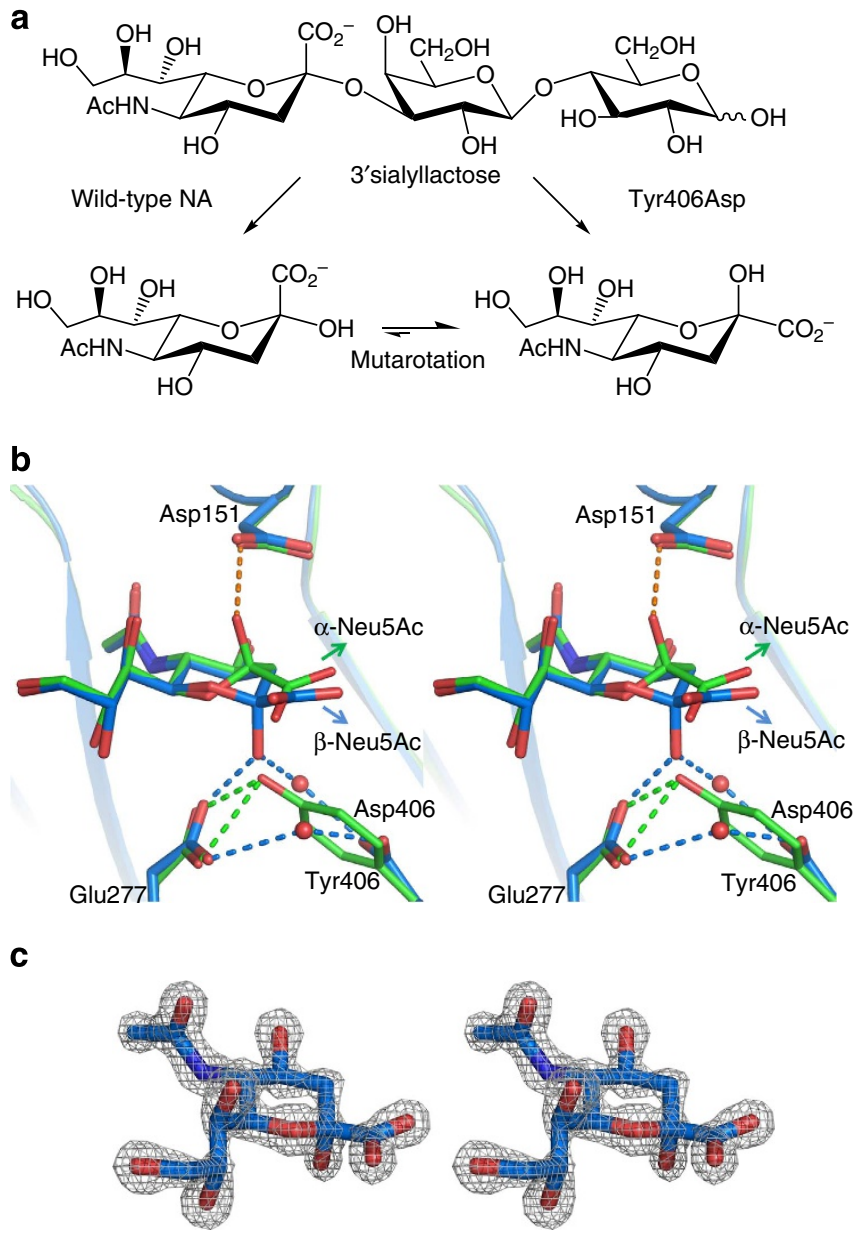

Figure 2 | N2-Tyr406Asp operates via an inverting sialidase mechanism. (a) Scheme outlining the initial formation of $\alpha$-Neu5Ac by wild-type influenza NA and initial formation of $\beta-N e u 5 A c$ by influenza NA with a Tyr406Asp substitution. (b) Stereo illustration of the novel N2-Tyr406Asp$\beta$-Neu5Ac complex (marine) in comparison to wild-type A/Tokyo/3/ 1967(H2N2) N2 complexed with $\alpha$-Neu5Ac (green) (PDB ID: 2BAT). Two additional water molecules present in the N2-Tyr406Asp- $\beta$-Neu5Ac active site are coloured cyan. (c) Stereo illustration of $\beta$-Neu5Ac when bound to the N2-Tyr406Asp active site with the 2|Fol-|Fc|map contoured at $2.0 \sigma$. 
leaving group can face toward the outside of the NA active site cavity and depart easily (Fig. 3a). Therefore, 2 $\alpha$,3ax-difluoro-Neu5Ac was our primary target for a potential influenza virus NA covalent inhibitor.

$2 \alpha, 3 a x$-difluoro-Neu5Ac is a potent influenza virus inhibitor. $2 \alpha, 3$ ax-difluoro-Neu5Ac indeed displayed potent in vitro NA inhibition, using both $\mathrm{rN} 2$ and live A/Jiangxi/262/2005(H3N2) virus, with $\mathrm{IC}_{50}$ values of 201 and $286 \mathrm{nM}$, respectively (Table 3 ). Inhibition of viral replication of seasonal A/Aichi/102/ 2008(H3N2) and A/Aichi/75/2008(H3N2) by $2 \alpha, 3$ ax-difluoroNeu5Ac in an AX4 cell-based assay was even more potent, both with $\mathrm{IC}_{50}$ values of $16.1 \mathrm{nM}$ (Table 3). $2 \alpha$,3ax-difluoro-Neu5Ac was also a highly potent low nanomolar level inhibitor of B/Lee/ 40 influenza B virus (Table 3, Fig. 4). Although no dramatic time dependent effects on influenza A virus NA inhibition were observed when using submicromolar concentrations of $2 \alpha, 3 \mathrm{ax}-$ difluoro-Neu5Ac, inhibition of NA from B/Lee/40 resulted in more pronounced time-dependent effects, even at concentrations of 10-100 nM (Fig. 4).

In a previous mass spectrometric based analysis, binding of zanamivir and Neu5Ac2en to an 09N1 peptide containing Tyr406 was successfully detected ${ }^{31}$. Furthermore, covalent modification of the conserved active-site tyrosine was also detected in Trypanosoma rangeli sialidase using mass spectrometric methods ${ }^{26}$. Despite our attempts, we were unable to detect the covalent intermediate using similar methods; however, the observation of time-dependent inhibition indicates that the covalent intermediate is being formed in solution with influenza NA.

One major concern for the use of fluorinated covalent inhibitors is toxicity; however, we observed no cytotoxity to

\begin{tabular}{|c|c|c|}
\hline & N2-covalent & $\begin{array}{c}\text { N2-Tyr406Asp- } \\
\text { B-Neu5Ac }\end{array}$ \\
\hline \multicolumn{3}{|l|}{ Data collection } \\
\hline Space group & $\mathrm{C} 2221$ & P21 \\
\hline \multicolumn{3}{|l|}{ Cell dimensions } \\
\hline$a, b, c(\AA)$ & $114.70,139.70,140.07$ & $83.22,114.83,84.26$ \\
\hline$\alpha, \beta, \gamma\left({ }^{\circ}\right)$ & $90,90,90$ & $90,99.7,90$ \\
\hline Resolution $(\AA)$ & $50-1.80(1.86-1.80)$ & $50-1.50(1.55-1.50)$ \\
\hline$R_{\text {merge }}$ & $0.101(0.385)$ & $0.098(0.367)$ \\
\hline$|/ \sigma|$ & $19.6(4.9)$ & $15.9(3.7)$ \\
\hline Completeness (\%) & $100.0(100.0)$ & $99.9(99.8)$ \\
\hline Redundancy & $7.4(7.5)$ & $4.6(4.6)$ \\
\hline \multicolumn{3}{|l|}{ Refinement } \\
\hline Resolution $(\AA)$ & $38.82-1.80$ & $39.05-1.50$ \\
\hline No. reflections & 103136 & 248190 \\
\hline$R_{\text {work }} / R_{\text {free }}$ & $0.1513 / 0.1749$ & $0.1400 / 0.1613$ \\
\hline \multicolumn{3}{|l|}{ No. atoms } \\
\hline Protein & 6235 & 12485 \\
\hline Ligand/ion & 44 & 162 \\
\hline Water & 927 & 2189 \\
\hline \multicolumn{3}{|l|}{$B$-factors } \\
\hline Protein & 17.5 & 10.4 \\
\hline Ligand/ion & 15.6 & 14.7 \\
\hline Water & 33.2 & 23.7 \\
\hline \multicolumn{3}{|l|}{ R.M.S. deviations } \\
\hline Bond lengths $(\AA)$ & 0.007 & 0.007 \\
\hline Bond angles $\left(^{\circ}\right)$ & 1.199 & 1.366 \\
\hline
\end{tabular}

MDCK cells when using up to $1 \mathrm{mM} 2 \alpha, 3$ ax-difluoro-Neu5Ac as determined by an MTT assay. Interestingly, $2 \beta, 3$ eq-difluoroNeu5Ac was a better inhibitor of rN2-Tyr406Asp than wild-type

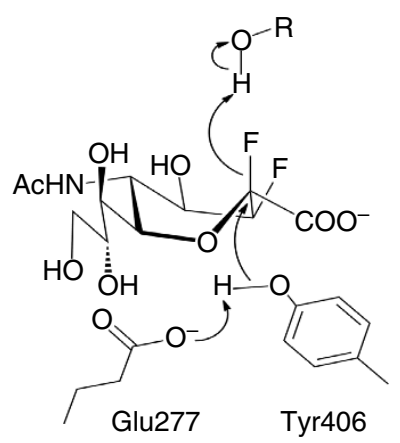

b

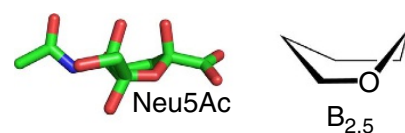

Covalent complex
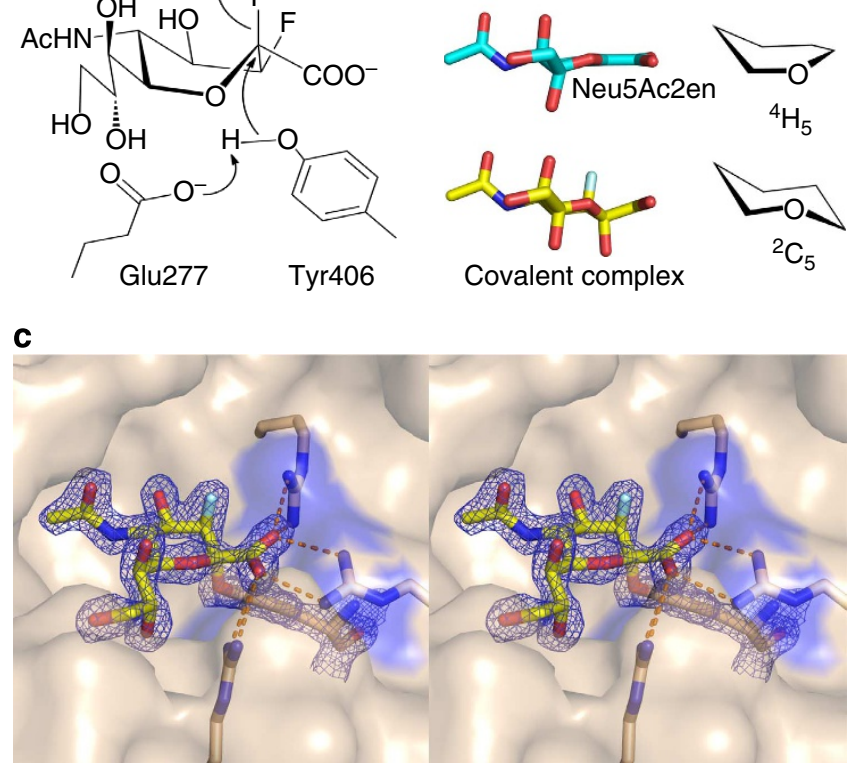

Figure 3 | Structure-based design and analysis of the first influenza NA covalent inhibitor. (a) Scheme for the mechanism-based design of covalent inhibition targeting influenza NA using 2 $\alpha$,3ax-difluoro-Neu5Ac. (b) Neu5Ac (green) adopts a boat conformation after binding to NA active site (PDB ID: 2BAT). Neu5Ac2en (cyan), a putative oxocarbenium ion transition state analogue, adopts a half-chair conformation(PDB ID: 1F8B). The covalently bound 3ax-fluoro-Neu5Ac moiety (yellow) adopts a chair conformation where $\mathrm{C}-2$ moves toward Tyr 406. This illustrates that in order to arrive at a covalently-linked intermediate, the reaction trajectory must still involve a planar intermediate. (c) Stereo illustration of the first influenza NA covalent complex, formed between rN2 Tyr406 (wheat) and a 3ax-fluoro-Neu5Ac moiety (yellow). The 2|Fol-|Fc|map for the 3ax-fluoroNeu5Ac moiety and Tyr406 is shown contoured at $2.0 \sigma$.

Table 3 | 2 $\alpha$,3ax-difluoro-Neu5Ac is a potent inhibitor of influenza virus NA and influenza virus replication.

\begin{tabular}{|c|c|c|}
\hline & $I C_{50}(n M)$ & $95 \% \mathrm{Cl}(\mathrm{nM})$ \\
\hline rN2-Tyr406Asp* & $>20 \times 10^{6}$ & 一 \\
\hline$r N 2 *$ & 201 & $179-226$ \\
\hline A/Jiangxi/262/2005(H3N2)* & 286 & $271-303$ \\
\hline A/Aichi/102/2008† & 16.1 & $10.5-24.6$ \\
\hline A/Aichi/75/2008† & 16.1 & $7.67-33.7$ \\
\hline r09N1* & 226 & $196-261$ \\
\hline r09N1-His274Tyr* & 247 & $233-261$ \\
\hline B/Lee/40* & 56.8 & $50.9-63.4$ \\
\hline B/Lee/40\$ & 9.85 & $4.30-22.6$ \\
\hline \multicolumn{3}{|c|}{$\begin{array}{l}\text { Inhibition of NA activity from rN2, rN2-Tyr406Asp, A/Jiangxi/262/2005(H3N2), r09N1, } \\
\text { r09N1-His274Tyr and B/Lee/40 by } 2 \alpha, 3 a x-d i f l u o r o-N e u 5 A c \text {. Inhibition of A/Aichi/102/ } \\
2008(H 3 N 2) \text { and A/Aichi/75/2008(H3N2) viral replication in AX4 cells by } 2 \alpha, 3 a x-d i f l u o r o- \\
\text { Neu5Ac. MDCK cell-based inhibition of B/Lee/40 viral replication by } 2 \alpha, 3 a x-d i f l u o r o-N e u 5 A c \text { in } \\
\text { a plaque-reduction assay. } \\
\text { *Inhibition of NA enzymatic activity. } \\
\text { †Inhibition of viral replication in an AX4 cell-based assay. \$Inhibition of viral replication in a } \\
\text { MDCK cell-based plaque-reduction assay. }\end{array}$} \\
\hline
\end{tabular}


a
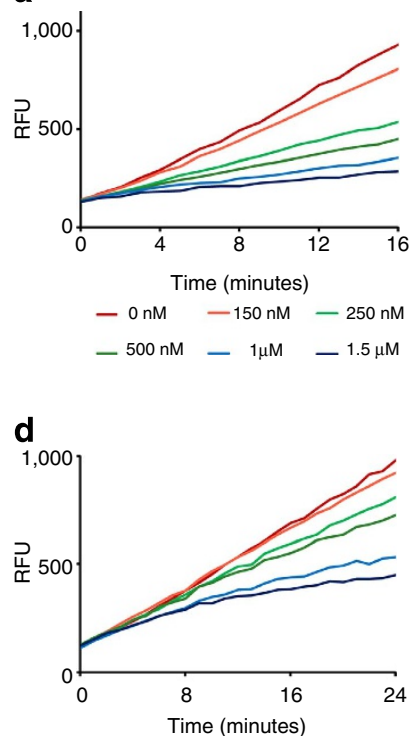

$-0 \mathrm{nM} \quad-150 \mathrm{nM}-250 \mathrm{nM}$

$-500 \mathrm{nM}-1 \mu \mathrm{M}-1.5 \mu \mathrm{M}$ b

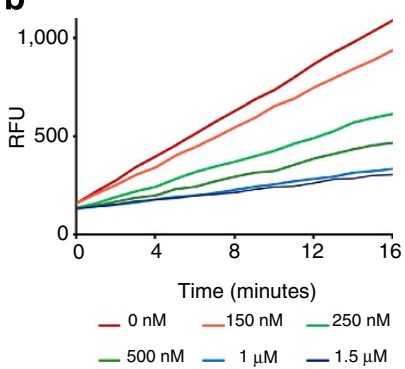

e

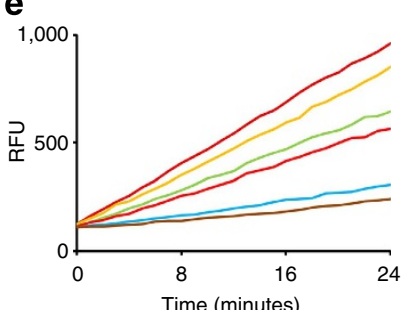

$\begin{aligned} & 0 \mathrm{nM} \\ & -50 \mathrm{nM}\end{aligned}-25 \mathrm{nM}$

$-50 \mathrm{nM} \quad-150 \mathrm{nM} \quad-300 \mathrm{nM}$

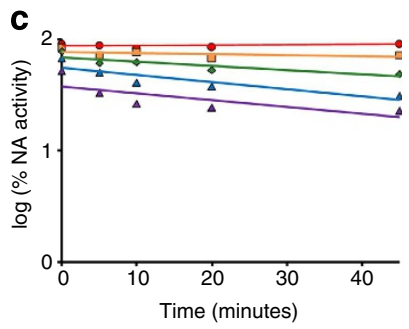

$\rightarrow 50 \mu \mathrm{M} \rightarrow 200 \mathrm{nM} \nrightarrow 500 \mathrm{nM}$

$$
\rightarrow 1 \mu \mathrm{M} \quad \rightarrow 2 \mu \mathrm{M}
$$

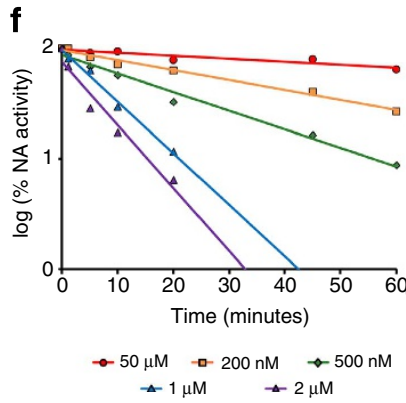

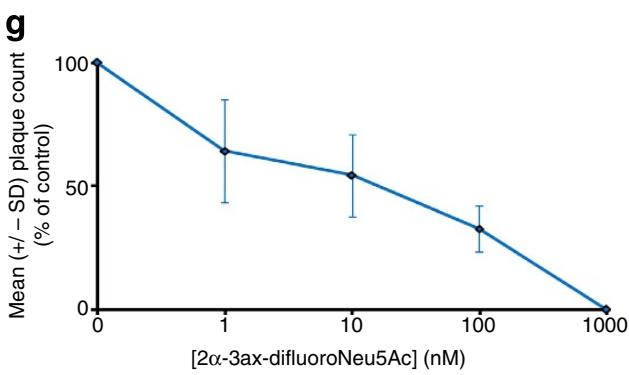

Figure 4 | Inhibition of A/Jiangxi/262/2005(H3N2) and B/Lee/40 influenza viruses. (a) Inhibition of A/Jiangxi/262/2005(H3N2) NA activity using no preincubation of virus with $0-1.5 \mu \mathrm{M} 2 \alpha, 3$ ax-difluoro-Neu5Ac (final concentration). (b) Inhibition of A/Jiangxi/262/2005(H3N2) NA activity using 30 min preincubation of virus with $0-1.5 \mu \mathrm{M} 2 \alpha$,3ax-difluoro-Neu5Ac (final concentration). (c) Plot of $\log$ [\% H3N2 NA activity] versus incubation time for different preincubation concentrations of $2 \alpha, 3$ ax-difluoro-Neu5Ac. (d) Inhibition of B/Lee/40 NA activity using no preincubation of virus with $0-1.5 \mu M$ $2 \alpha, 3 a x$-difluoro-Neu5Ac (final concentration). (e) Inhibition of B/Lee/40 NA activity using 20 min preincubation of virus with 0-300 nM 2 $\alpha, 3 a x-d i f l u o r o-$ Neu5Ac (final concentration). (f) Plot of $\log [\% \mathrm{~B} /$ Lee/40 NA activity] versus incubation time for different preincubation concentrations of $2 \alpha, 3 a x-d i f l u o r o-$ Neu5Ac. (g) Inhibition of B/Lee/40 plaque formation by $1 \mathrm{nM}, 10 \mathrm{nM}, 100 \mathrm{nM}$ and $1 \mu \mathrm{M} 2 \alpha, 3$ ax-difluoro-Neu5Ac. Each point with error bars represents the mean \% plaque count (based upon 4 values for $1 \mathrm{nM}$ and 6 values for $0 \mathrm{nM}, 10 \mathrm{nM}, 100 \mathrm{nM}$ and $\mu \mathrm{M}$ ) \pm s.d.

rN2 (2 mM 2ß,3eq-difluoro-Neu5Ac produced 54\% inhibition of rN2-Tyr406Asp and only $25 \%$ inhibition of wild-type rN2). This can be explained by the rN2-Tyr406Asp- $\beta$-Neu5Ac complex structure, where the additional space resulting from the substitution of tyrosine with aspartic acid can better accommodate 23,3eq-difluoro-Neu5Ac. The poor inhibition of rN2-Tyr406Asp by $2 \alpha, 3$ ax-difluoro-Neu5Ac ( $>20 \mathrm{mM}$, Table 3 ) further indicated that it targets Tyr406 as a covalent inhibitor in solution.

Probing the influenza virus NA covalent intermediate. To determine if a covalent complex is really formed between influenza virus NA and $2 \alpha, 3$ ax-difluoro-Neu5Ac, quality rN2 protein crystals were soaked with inhibitor and the complex crystal structure was solved at $1.80 \AA$. A N2-covalent complex structure was successfully obtained, with clear electron density revealing a $1.46 \AA$ covalent bond between the Tyr406 terminal oxygen and the C-2 of a 3ax-fluoro-Neu5Ac moiety (Fig. 3c). The 3axfluoro-Neu5Ac moiety carboxylate is shifted closer toward Arg118 and away from Arg292 relative to N2 complexed with Neu5Ac. As expected, the C-2 fluoride leaving group is not present, confirming that it has departed during the formation of the molecular bond with Tyr406. Although no method is readily available to directly detect a naturally occurring covalent NA intermediate, the ability to obtain the influenza NA covalent complex using $2 \alpha, 3$ ax-difluoro-Neu5Ac still suggests that a covalently bound intermediate is a critical step during hydrolysis of natural NA substrates. Therefore, we propose that influenza NA follows a double-displacement mechanism similar to the classical retaining glycosidase mechanism $^{16}$.

The covalently linked 3ax-fluoro-Neu5Ac moiety adopts a chair conformation, whereas $\alpha$-Neu5Ac and Neu5Ac2en adopt boat and half-chair conformations ${ }^{10}$, respectively, when bound to influenza NA (Fig.3b). In our N2-covalent complex, the unique C-3 fluorine of the 3ax-fluoro-Neu5Ac moiety is $3.51-3.55 \AA$ away from the closest neighbouring residue Arg118. Aside from the differences regarding C-2 due to a novel covalent bond and chair conformation, the remaining interactions between the $3 \mathrm{ax}-$ fluoro-Neu5Ac moiety and influenza NA highly resemble those of $\alpha-\mathrm{Neu} 5 \mathrm{Ac}$ and Neu5Ac2en. 
Effective covalent inhibition of oseltamivir-resistant NA. The similarity of the 3ax-fluoro-Neu5Ac moiety binding mode to the natural NA ligand Neu5Ac is a strong indication that the covalent inhibitor $2 \alpha, 3$ ax-difluoro-Neu5Ac should be effective against drug-resistant NA. This is also illustrated by the high similarity between the binding modes of the covalent inhibitor and zanamivir, which remain effective against oseltamivir-resistant His274Tyr $\mathrm{NA}^{32}$ (Fig. 5). Therefore, we also tested inhibition against oseltamivir-resistant rN1-His274Tyr from the 2009 H1N1 pandemic in comparison to wild-type rN1. Although His274Tyr $\mathrm{N} 1$ has been reported to exhibit more than 200-times lower inhibition by oseltamivir than by wild-type $\mathrm{N}^{32}$, the covalent inhibitor 2 $\alpha, 3$ ax-difluoro-Neu5Ac inhibited recombinant 2009 pandemic N1 (r09N1-His274Tyr) at the same level as wild-type recombinant 2009 pandemic N1 (r09N1) (Table 3). This demonstrates that $2 \alpha, 3$ ax-difluoro-Neu $5 \mathrm{Ac}$ is an effective inhibitor against the most common oseltamivir-resistant NA.

\section{Discussion}

In this study, we confirm that the conserved active site Tyr406 has an integral role in the catalytic mechanism of influenza virus NA and that Tyr406 can be successfully targeted for the design of covalent inhibitors. Furthermore, due to its importance in the catalytic cycle, drug-resistant mutations leading to substitution of Tyr406 are extremely unfavourable for the influenza virus. This is further illustrated by the fact that Tyr406 is almost $100 \%$ conserved among all influenza virus NA proteins. Therefore, the targeting of Tyr406 with covalent inhibitors is a next-generation strategy for influenza inhibitor development, which is highly advantageous in terms of drug resistance.

However, the first generation of influenza NA competitive inhibitors are still highly vulnerable to drug resistance. Long before the widespread circulation of natural oseltamivir-resistant influenza virus strains, it had been proposed that inhibitors that

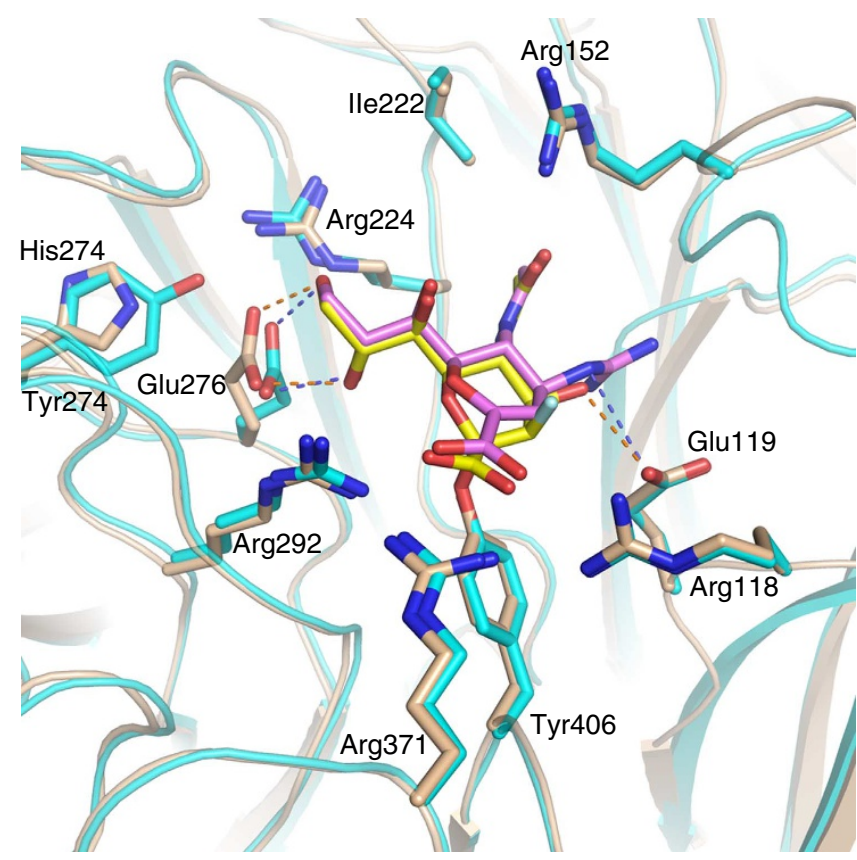

Figure 5 | Structural comparison of the N2-covalent complex with the oseltamivir-resistant N1-His274Tyr zanamivir complex. The binding mode of the 3ax-fluoro-Neu5Ac moiety (yellow) in N2 (wheat) and zanamivir (violet) in N1-His274Tyr (cyan) (PDB ID: 3CKZ) is highly similar, indicating that $2 \alpha$,3ax-difluoro-Neu5Ac should remain effective against common oseltamivir-resistant influenza viruses. more closely resemble natural NA ligands are less likely to develop drug resistance ${ }^{33}$. Oseltamivir contains a hydrophobic pentyl ether group in place of the glycerol moiety of the natural NA ligand Neu5Ac and therefore oseltamivir resistance is easily developed. Upon binding of natural NA ligands, Glu276 hydrogen bonds with the ligand glycerol moiety. However, in order to accommodate oseltamivir, Glu276 must rotate to form a salt bridge with Arg224. The most common oseltamivir-resistant mutations, like His274Tyr, interfere with the rotation of Glu276, which is necessary to accommodate oseltamivir ${ }^{32}$.

Although zanamivir resistance is not as common as oseltamivir resistance due to a higher similarity with Neu5Ac, amino-acid substitutions, like Glu136Lys, can still arise that interfere with the binding of the zanamivir C-4 guanidino group. Aside from the similarity with the natural NA ligand, Neu5Ac, zanamivir is also not as commonly used as oseltamivir, which contributes to the lower frequency of zanamivir-resistant viruses. Nevertheless, the oseltamivir hydrophobic pentyl ether and the zanamivir C-4 guanidino that contribute to drug resistance are the key modifications that contribute to their potency. Therefore, $2 \alpha, 3$ ax-difluoro-Neu5Ac, which more closely resembles the natural NA ligand, can still exhibit highly potent inhibition due to its action as a covalent inhibitor, and thus possesses significant advantages.

Still, rational modification of $2 \alpha, 3$ ax-difluoro-Neu5Ac should be vigorously pursued to produce more potent next-generation covalent influenza virus NA inhibitors. As we observe stronger time-dependent inhibition (Fig. 4) in combination with a significant increase in potency (Table 3) for influenza B NA, the increased time dependence must be a result of an increased lifetime of a reversible covalent intermediate rather than an effect of slow inhibitor binding ${ }^{34}$. Therefore, this illustrates that further stabilization of the covalent intermediate is a promising strategy to improve the effectiveness of next-generation covalent influenza virus NA inhibitors.

Two major strategies can be taken for the stabilization of the influenza NA Tyr406-inhibitor covalent bond: modification of the covalent inhibitor to disrupt steric effects imposed by the NA active site that destabilize the favored chair conformation of the covalent intermediate, and modification of the covalent inhibitor to further destabilize build-up of positive charge around C-2, thereby stabilizing the covalent intermediate.

In regards to the first strategy, it is difficult to predict from our complex structure, what specific modifications will stabilize the chair conformation of the covalent intermediate. In regards to the second strategy, a stronger C-3 electron-withdrawing group might increase the lifetime of the covalent intermediate. As observed in the N2-covalent complex structure, there is no significant interaction between the 3-ax-fluoro-Neu5Ac moiety 3-ax-fluorine with influenza N2, indicating that the C-3 fluorine contributes to the inhibition potency by stabilization of the covalent intermediate. The lack of interactions between the 3-ax-fluorine and the NA active site should also allow for sufficient room to accommodate a bulkier, stronger C-3 electronwithdrawing group that may further stabilize the NA covalent intermediate. Although the closed conformation of the 150-loop in group 2 NA structures as well as the 2009 pandemic N1 ${ }^{40}$ could potentially hinder the binding of a bulkier C-3 group, recent studies have demonstrated that the 150-loop is inherently flexible $^{35}$ and, therefore, is able to accommodate bulkier electronwithdrawing groups.

In conclusion, this study contributes the first proof-of-concept for the use of covalent influenza NA inhibitors as a true nextgeneration strategy for influenza drug development. The firstgeneration influenza NA inhibitors, zanamivir and oseltamivir, are regarded as a classical example of structure-based competitive 
inhibitor design; however, novel strategies for NA inhibition has remained limited. The first covalent influenza NA-complex offered here provides a framework for the rational and structure-based development of highly potent next-generation influenza virus inhibitors of the future.

\section{Methods}

Cells and viruses. AX4 cells, MDCK cells with increased levels of sialyl- $\alpha 2,6-$ galactose moieties, were grown and maintained following previous protocols ${ }^{36,37}$ The seasonal influenza virus isolates A/Aichi/102/2008(H3N2), A/Aichi/75/ 2008(H3N2) (Aichi Pref. Inst. Public Health, Japan), A/Jiangxi/262/2005(H3N2) (China CDC) and recombinant B/Lee/40 were amplified in MDCK cells. Viral culture supernatants were harvested, concentrated and stored at $-80^{\circ} \mathrm{C}$.

Expression and purification of influenza NAs. Recombinant NAs were purified to homogeneity after being expressed in a baculovirus expression system, based on the original method by Xu et al. ${ }^{38-41}$ with modifications. For rN2-Tyr406Asp and 2009 pandemic rN1-His274Tyr, point mutations were introduced by site-directed mutagenesis based upon the corresponding wild-type NAs (Generay, China).

Enzymatic kinetics. The activity of rN2 and rN2-Tyr406Asp was monitored using a fluorometric assay based on methods from Potier et al. ${ }^{42} \mathrm{NA}$ activity was monitored using 0-500 $\mu \mathrm{M} 4$-methylumbelliferyl-Neu5Ac (J\&K Scientific) with 33 $\mathrm{mM}$ MES and $4 \mathrm{mM} \mathrm{CaCl}_{2}$ ( $\mathrm{pH}$ 6.0). The final concentration of enzymes used for wild-type rN2 and rN2-Tyr406Asp were 1 and $167 \mathrm{nM}$, respectively. Reactions were performed in a total volume of $50 \mu \mathrm{l}$ at $37^{\circ} \mathrm{C}$, and monitored every $30 \mathrm{~s}$ over the course of $30 \mathrm{~min}$ on a SpectraMax M5 (Molecular Devices) at excitation and emission wavelengths of 355 and $460 \mathrm{~nm}$, respectively. All assays were performed in no less than triplicates and the final data were fitted to the Michaelis-Menten equation using GraphPad Prism to determine the Michaelis-Menten constant $\left(K_{\mathrm{m}}\right)$ and maximum velocity of substrate conversion $\left(V_{\max }\right)$.

In vitro NA inhibition assay. A 4-methylumbelliferyl-Neu5Ac-based fluorometric NA assay was also used to test competitive NA inhibition ${ }^{42}$. Recombinant NA or live influenza virus was diluted until NA activity was in the appropriate range for fluorescence detection of liberated 4-methylumbelliferone on a SpectraMax M5 (Molecular Devices), using excitation and emission wavelengths of 355 and $460 \mathrm{~nm}$, respectively. 4-methylumbelliferyl-Neu5Ac was used at a final concentration of $100 \mu \mathrm{M}$. Five concentrations of $0-5 \mu \mathrm{M} 2 \alpha$,3ax-difluoro-Neu5Ac and a positive control with an equivalent volume of PBS were used for each replicate. For quantification of the B/Lee/40 half maximal inhibitory concentration $\left(\mathrm{IC}_{50}\right), 2 \alpha, 3$ ax-difluoro-Neu5Ac was preincubated with live $\mathrm{B} / \mathrm{Lee} / 40$ virus for 20 min before starting the reaction. For influenza A NA, all components were mixed simultaneously at the start of the reaction. Each assay was done in no less than triplicates. $\mathrm{IC}_{50}$ values were determined by plotting log[inhibitor] versus per cent inhibition using the sigmoidal dose response (variable slope) function in GraphPad Prism.

${ }^{\mathbf{1}}$ H NMR NA activity assay. ${ }^{1} \mathrm{H}$ NMR spectroscopy (600 MHz Bruker DXM-600, Bruker, CAS Institute of Biophysics) was employed to monitor the hydrolysis of $3^{\prime}$-sialyllactose (Carbosynth) catalysed by NA until the reactions reached equilibrium. For each reaction, a total volume of $500 \mu \mathrm{l}$ with the ratio of $\mathrm{D}_{2} \mathrm{O}$ to $\mathrm{H}_{2} \mathrm{O}$ of 1:9 was used, containing enzyme, $2 \mathrm{mM}$ substrate, $20 \mathrm{mM}$ phosphate, $100 \mathrm{mM}$ $\mathrm{NaCl}$ and $1 \mathrm{mM} \mathrm{CaCl}_{2}$ (pH 6.0). The final concentrations for wild-type $\mathrm{rN} 2$ and rN2-Tyr406Asp were $32 \mathrm{nM}$ and $13 \mu \mathrm{M}$, respectively. Spectral data were collected at $37^{\circ} \mathrm{C}$ with 64 scans and a relaxation delay of $2 \mathrm{~s}$. An initial spectrum containing only substrate and buffer (without NA) was acquired and referred to as time zero. The same reactions were also performed at $25^{\circ} \mathrm{C}$ for monitoring and no obvious difference was seen.

Crystallization, soaking and data collection. The baculovirus expression system allows for the production of high quantities of soluble NA for crystallization experiments; however, despite our recent success with the structural characterization of $09 \mathrm{~N} 1$ (ref. 40), we were unable to obtain enough quality N1 crystals for the soaking experiments carried out in this study. Instead, quality rN2 crystals were obtained as previously described ${ }^{41}$, and rN2-Tyr406Asp crystals were grown by vapour diffusion in hanging drops that consist of $1 \mu$ l concentrated protein $\left(6 \mathrm{mg} \mathrm{ml}^{-1}\right)$ and $1 \mu \mathrm{l}$ reservoir solution $(0.1 \mathrm{M} \mathrm{MES} \mathrm{monohydrate} \mathrm{pH} 6.0,14 \% \mathrm{w} / \mathrm{v}$ polyethylene glycol 4000). rN2 and rN2-Tyr406Asp crystals were soaked in the corresponding mother liquor containing $10 \mathrm{mM}$ of $2 \alpha, 3 \mathrm{ax}$-difluoro-Neu5Ac2 at $4^{\circ} \mathrm{C}$ for $45 \mathrm{~min}$ and $10 \mathrm{mM} 3^{\prime}$-sialyllactose at $18^{\circ} \mathrm{C}$ for $180 \mathrm{~min}$, respectively. After flash-cooling at $100 \mathrm{~K}$ with cryoprotection, diffraction data were collected at SSRF beamline BL17U and KEK beamline BL17A for the N2-Tyr406Asp- $\beta$-Neu5Ac complex and N2-covalent complex, respectively.
Data processing and structure solution. Diffraction data were processed and scaled using HKL2000 (ref. 43) and the statistics are outlined in Table 2. The structure of N2-Tyr406Asp complexed with $\beta$-Neu5Ac and the wild-type N2covalent complex were both determined by molecular replacement using $\mathrm{Phaser}^{44}$ from the CCP4 suite ${ }^{45}$ with the structure of A/Tokyo/3/1967(H2N2)N2 (PDB ID: 1IVG) as the search model. REFMAC5 (ref. 46) and COOT ${ }^{47}$ were employed for initial restrained rigid-body refinement and model building, respectively. Further cycles of refinement were carried out with phenix.refine implemented in the Phenix package ${ }^{48}$. The stereochemical quality of the obtained model was finally assessed using Procheck ${ }^{49}$.

Cytotoxicity assay. Cytotoxicity was assessed in MDCK cells following a standard MTT assay protocol ${ }^{50}$

AX4 cell-based virus inhibition assay. Inhibition of influenza virus propagation was measured using AX4 cells in the presence of serial dilutions of $2 \alpha, 3 \mathrm{ax}$-difluoroNeu5Ac $(0.1-100 \mu \mathrm{M})^{37,51}$. Influenza virus at a multiplicity of infection of 0.03 was preincubated with a medium containing $2 \mu \mathrm{g} \mathrm{ml}^{-1}$ acetylated trypsin in the absence or presence of serially diluted inhibitor for $1 \mathrm{~h}$ at $4{ }^{\circ} \mathrm{C}$. Then, the inhibitorvirus mixture was added onto the AX4 cell monolayers, which were incubated for $18-20 \mathrm{~h}$ at $37^{\circ} \mathrm{C}$. Infected cells were fixed and permealized with methanol followed by staining with antiviral NP mouse antibody and $\beta$-galactosidase-conjugated antimouse IgG. A substrate solution containing 4 -methylumbelliferyl- $\beta$-D-galactoside and $\mathrm{MgCl}_{2}$ was added to initiate the galactosidase reaction. 4-methylumbelliferyl was detected with an excitation wavelength of $355 \mathrm{~nm}$ and an emission wavelength of $460 \mathrm{~nm}$ on a Mithras LB940 microplate reader (Berthold Technologies, Pforzheim, Germany) to determine the virus titre in the infected cells.

The infectious foci were also observed using a peroxidase-based chromogenic assay. The cells were treated with HRP-conjugated goat anti-mouse IgG antibody and influenza virus was stained blue by incubation with $\mathrm{H}_{2} \mathrm{O}_{2}, N$,N-diethyl-pphenylenediamine dihydrochloride and 4-chloro-1-naphthol. $\mathrm{EC}_{50}$ values were calculated graphically with GraphPad Prism using the fluorescent assay data. Eleven replicates were used for A/Aichi/102/2008 H3N2 and six replicates were used for A/Aichi/75/2008 H3N2.

Plaque-reduction assay. The effectiveness of $2 \alpha, 3 \mathrm{ax}$-difluoro- $N$-acetylneuraminic acid against influenza plaque reduction was assessed with recombinant B/Lee/40 influenza virus based on a previously reported method ${ }^{52}$. MDCK cell monolayers were inoculated with virus $(\sim 40$ PFU per well) and the plates were incubated for $1 \mathrm{~h}$ at $37^{\circ} \mathrm{C}$. The inoculum was then removed and an agarose overlay containing appropriate inhibitor dilution was added. After 2 days of incubation at $37^{\circ} \mathrm{C}$, the results of plaques reduction were recorded, and the $\mathrm{EC}_{50}$ values were calculated graphically using GraphPad Prism. Six replicates were used for the plaquereduction assay.

\section{References}

1. Imai, M. et al. Experimental adaptation of an influenza H5 HA confers respiratory droplet transmission to a reassortant $\mathrm{H} 5 \mathrm{HA} / \mathrm{H} 1 \mathrm{~N} 1$ virus in ferrets. Nature 486, 420-428 (2012).

2. Herfst, S. et al. Airborne transmission of influenza A/H5N1 virus between ferrets. Science 336, 1534-1541 (2012).

3. Guan, Y. et al. The emergence of pandemic influenza viruses. Protein Cell 1, 9-13 (2010).

4. Gao, G. F. \& Sun, Y. It is not just AIV: from avian to swine-origin influenza virus. Sci. China Life Sci. 53, 151-153 (2010)

5. Jagger, B. W. et al. An overlapping protein-coding region in influenza A virus segment 3 modulates the host response. Science 337, 199-204 (2012).

6. Vavricka, C. J. et al. Special features of the 2009 pandemic swine-origin influenza A H1N1 hemagglutinin and neuraminidase. Chin. Sci. Bull. 56, 1747-1752 (2011).

7. Sun, Y. et al. In silico characterization of the functional and structural modules of the hemagglutinin protein from the swine-origin influenza virus A (H1N1)-2009. Sci. China Life Sci. 53, 633-642 (2010).

8. von Itzstein, M. Influenza Virus Sialidase-A Drug Discovery Target (Springer, Basel, 2012).

9. Centers for Disease Control and Prevention. Influenza activity-United States and worldwide, 2007-08 season. Morb. Mortal. Wkly. Rep. 58, 115-119 (2009).

10. von Itzstein, M. et al. Rational design of potent sialidase-based inhibitors of influenza virus replication. Nature 363, 418-423 (1993).

11. Kim, C. U. et al. Influenza neuraminidase inhibitors possessing a novel hydrophobic interaction in the enzyme active site: design, synthesis, and structural analysis of carbocyclic sialic acid analogues with potent antiinfluenza activity. J. Am. Chem. Soc. 119, 681-690 (1997).

12. Yamashita, M. et al. CS-8958, a prodrug of the new neuraminidase inhibitor R-125489, shows long-acting anti-influenza virus activity. Antimicrob. Agents Chemother. 53, 186-192 (2009). 
13. Chong, A. K., Pegg, M. S., Taylor, N. R. \& von Itzstein, M. Evidence for a sialosyl cation transition-state complex in the reaction of sialidase from influenza virus. Eur. J. Biochem. 207, 335-343 (1992).

14. Taylor, N. R. \& von Itzstein, M. Molecular modeling studies on ligand binding to sialidase from influenza virus and the mechanism of catalysis. J. Med. Chem. 37, 616-624 (1994).

15. Rye, C. S. \& Withers, S. G. Glycosidase mechanisms. Curr. Opin. Chem. Biol. 4, 573-580 (2000).

16. Guo, X., Laver, W. G., Vimir, E. \& Sinnott, M. L. Catalysis by two sialidases with the same protein fold but different stereochemical courses: a mechanistic comparison of the enzymes from influenza A virus and Salmonella typhimurium. J. Am. Chem. Soc 116, 5572-5578 (1994).

17. Watson, J. N., Dookhun, V., Borgford, T. J. \& Bennet, A. J. Mutagenesis of the conserved active-site tyrosine changes a retaining sialidase into an inverting sialidase. Biochemistry 42, 12682-12690 (2003).

18. Watts, A. G., Oppezzo, P., Withers, S. G., Alzari, P. M. \& Buschiazzo, A. Structural and kinetic analysis of two covalent sialosyl-enzyme intermediates on Trypanosoma rangeli sialidase. J. Biol. Chem. 281, 4149-4155 (2006).

19. Chan, J. et al. Bacterial and viral sialidases: contribution of the conserved active site glutamate to catalysis. Biochemistry 51, 433-441 (2012).

20. Chou, D. T. H., Watson, J. N., Scholte, A. A., Borgford, T. J. \& Bennet, A. J. Effect of neutral pyridine leaving groups on the mechanisms of influenza type A viral sialidase-catalyzed and spontaneous hydrolysis reactions of $\alpha-\mathrm{D}-N$ acetylneuraminides. J. Am. Chem. Soc. 122, 8357-8364 (2000).

21. Burmeister, W. P., Henrissat, B., Bosso, C., Cusack, S. \& Ruigrok, R. W. H. Influenza B virus neuraminidase can synthesize its own inhibitor. Structure 1, 19-26 (1993).

22. Ghate, A. A. \& Air, G. M. Site-directed mutagenesis of catalytic residues of influenza virus neuraminidase as an aid to drug design. Europ. J. Biochem. 258, 320-331 (1998)

23. Thomas, A., Jourand, D., Bret, C., Amara, P. \& Field, M. J. Is there a covalent intermediate in the viral neuraminidase reaction? A hybrid potential freeenergy study. J. Am. Chem. Soc. 121, 9693-9702 (1999).

24. Xu, G. G. et al. Three Streptococcus pneumoniae sialidases: three different products. J. Am. Chem. Soc. 133, 1718-1721 (2011)

25. Lentz, M. R., Webster, R. G. \& Air, G. M. Site-directed mutation of the activesite of influenza neuraminidase and implications for the catalytic mechanism. Biochemistry 26, 5351-5358 (1987).

26. Watts, A. G. \& Withers, S. G. The synthesis of some mechanistic probes for sialic acid processing enzymes and the labeling of a sialidase from Trypanosoma rangeli. Can. J. Chem. 82, 1581-1588 (2004).

27. Nakajima, T., Hori, H., Ohrui, H., Meguro, H. \& Ido, T. Synthesis of $\mathrm{N}$-acetyl3-fluoro-neuraminic acids. Agr. Biol. Chem. Tokyo 52, 1209-1215 (1988).

28. Tsuchiya, T. Chemistry and developments of fluorinated carbohydrates. Adv. Carbohydr. Chem. Biochem. 48, 91-277 (1990).

29. Hagiwara, T., Kijimasuda, I., Ido, T., Ohrui, H. \& Tomita, K. Inhibition of bacterial and viral sialidases by 3 -fluoro- $N$-acetylneuraminic acid. Carbohydr. Res. 263, 167-172 (1994).

30. Ikeda, K. et al. $2 \beta, 3 \beta$-difluorosialic acid derivatives structurally modified at the C-4 position: synthesis and biological evaluation as inhibitors of human parainfluenza virus type 1. Carbohydr. Res. 339, 1367-1372 (2004).

31. Swaminathan, K. \& Downard, K. M. Anti-viral binding to influenza neuraminidase by MALDI mass spectrometry. Anal. Chem. 84, 3725-3730 (2012).

32. Collins, P. J. et al. Crystal structures of oseltamivir-resistant influenza virus neuraminidase mutants. Nature 453, 1258-1261 (2008).

33. Varghese, J. N. et al. Drug design against a shifting target: a structural basis for resistance to inhibitors in a variant of influenza virus neuraminidase. Structure 6, 735-746 (1998).

34. Singh, J., Petter, R. C., Baillie, T. A. \& Whitty, A. The resurgence of covalent drugs. Nat. Chem. Biol. 10, 307-317 (2011).

35. Amaro, R. E., Cheng, X., Ivanov, I., Xu, D. \& McCammon, J. A. Characterizing loop dynamics and ligand recognition in human- and avian-type influenza neuraminidases via generalized born molecular dynamics and end-point free energy calculations. J. Am. Chem. Soc. 131, $4702-4709$ (2009).

36. Hatakeyama, S. et al. Enhanced expression of an $\alpha 2,6$-linked sialic acid on MDCK cells improves isolation of human influenza viruses and evaluation of their sensitivity to a neuraminidase inhibitor. J. Clin. Microbiol. 43, 4139-4146 (2005).

37. Sriwilaijaroen, N. et al. Mechanisms of the action of povidone-iodine against human and avian influenza A viruses: its effects on hemagglutination and sialidase activities. Virol. J. 6, 124 (2009).

38. Xu, X., Zhu, X., Dwek, R. A., Stevens, J. \& Wilson, I. A. Structural characterization of the 1918 influenza virus H1N1 neuraminidase. J. Virol. 82, 10493-10501 (2008)
39. Zhang, W. et al. Crystal structure of the swine-origin A (H1N1) - 2009 influenza A virus hemagglutinin (HA) reveals similar antigenicity to that of the 1918 pandemic virus. Protein Cell 1, 459-467 (2010)

40. Li, Q. et al. The 2009 pandemic H1N1 neuraminidase N1 lacks the 150-cavity in its active site. Nat. Struct. Mol. Biol. 17, 1266-1268 (2010).

41. Vavricka, C. J. et al. Structural and functional analysis of laninamivir and its octanoate prodrug reveals group specific mechanisms for influenza NA inhibition. PLoS Pathog. 7, e1002249 (2011).

42. Potier, M., Mameli, L., Belisle, M., Dallaire, L. \& Melancon, S. B. Fluorometric assay of neuraminidase with a sodium (4-methylumbelliferyl- $\alpha-\mathrm{D}-N$ acetylneuraminate) substrate. Anal. Biochem. 94, 287-296 (1979).

43. Otwinowski, Z. \& Minor, W. Processing of X-ray diffraction data collected in oscillation mode. Method. Enzymol. 276, 307-326 (1997).

44. Read, R. J. Pushing the boundaries of molecular replacement with maximum likelihood. Acta Crystallogr. D Biol. Crystallogr. 57, 1373-1382 (2001).

45. Collaborative Computational Project, Number 4. The CCP4 suite: programs for protein crystallography. Acta Crystallogr. D Biol. Crystallogr. 50, 760-763 (1994).

46. Murshudov, G. N., Vagin, A. A. \& Dodson, E. J. Refinement of macromolecular structures by the maximum-likelihood method. Acta Crystallogr. D Biol. Crystallogr. 53, 240-255 (1997)

47. Emsley, P. \& Cowtan, K. Coot: model-building tools for molecular graphics. Acta Crystallogr. D Biol. Crystallogr. 60, 2126-2132 (2004).

48. Adams, P. D. et al. PHENIX: a comprehensive python-based system for macromolecular structure solution. Acta Crystallogr. D Biol. Crystallogr. 66, 213-221 (2010).

49. Laskowski, R. A., Macarthur, M. W., Moss, D. S. \& Thornton, J. M. Procheck - a program to check the stereochemical quality of protein structures. J. Appl. Crystallogr. 26, 283-291 (1993).

50. Mosmann, T. Rapid colorimetric assay for cellular growth and survival: application to proliferation and cytotoxicity assays. J. Immunol. Methods 65, 55-63 (1983).

51. Sriwilaijaroen, N. et al. Antiviral effects of Psidium guajava Linn. (guava) tea on the growth of clinical isolated $\mathrm{H} 1 \mathrm{~N} 1$ viruses: Its role in viral hemagglutination and neuraminidase inhibition. Antivir. Res. 94, 139-146 (2012).

52. Hayden, F. G., Cote, K. M. \& Douglas, Jr. R. G. Plaque inhibition assay for drug susceptibility testing of influenza viruses. Antimicrob. Agents Chemother. 17, 865-870 (1980)

\section{Acknowledgements}

Research in G.F.G.'s lab is supported by the National 973 project (Grant No. 2011CB504703) and the National Natural Science Foundation of China (NSFC, Grant No. 81021003). G.F.G. is a leading principal investigator of the NSFC Innovative Research Group. C.J.V. is supported by the Chinese Academy of Sciences Fellowship for Young International Scientists (Grant No. 2011Y2SA01) and the NSFC Research Fund for Young International Scientists (Grant No. 31250110214). H.K. is supported by a grant-in-aid from Japan Society for the Promotion of Science (Grant No. 17580092 and 19580120). Y.S. is supported by a grant from the MEXT COE project for private universities. We acknowledge Hongna Huang and Yanfang Zhang for help with protein production, and Shuhua Zhou (NMR facility at Institute of Biophysics, CAS) for help with NMR experiments. We thank Professor Yoshihiro Kawaoka for providing the plasmids used to rescue B/Lee/40 influenza B virus and the AX4 cells used in this study. Finally, we are grateful to Hiroshi Ohrui (Yokohama College of Pharmacy, Yokohama, Japan) and Yoshitomo Suhara (Shibaura Institute of Technology, Tokyo, Japan) for their synthetic research of fluorinated sialic acid derivatives which contributed to this work.

\section{Author contributions}

C.J.V., Y.L., H.K., Y.S. and G.F.G. designed the research; C.J.V., Y.L., H.K., N.S., J.Q., K.T., Y.W., Q.L. and Y.L. performed the research; all authors analysed the data; and C.J.V., Y. L., and G.F.G. wrote the paper.

\section{Additional information}

Accession Code: Atomic coordinates and structure factors for the N2-Tyr406Asp$\beta$-Neu5Ac and N2-covalent complexes have been deposited in the Protein Data Bank under the accession codes $4 \mathrm{H} 53$ and $4 \mathrm{H} 52$, respectively.

Competing financial interests: The authors declare no competing financial interests.

Reprints and permission information is available online at http://npg.nature.com/ reprintsandpermissions/

How to cite this article: Vavricka, C.J. et al. Influenza neuraminidase operates via a nucleophilic mechanism and can be targeted by covalent inhibitors. Nat. Commun. 4:1491 doi: 10.1038/ncomms2487 (2013). 